\title{
Diabetes is associated with poor short-term prognosis in patients with hepatitis B virus-related acute-on-chronic liver failure
}

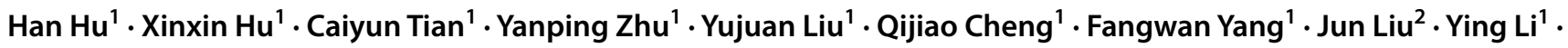 \\ Shide Lin $^{1}$ (1)
}

Received: 16 April 2021 / Accepted: 24 July 2021 / Published online: 10 August 2021

(c) Asian Pacific Association for the Study of the Liver 2021

\begin{abstract}
Background/purpose Associations between the disturbances in glucose homeostasis and prognosis in patients with hepatitis $B$ virus (HBV)-related acute-on-chronic liver failure (ACLF) remain unclear. This study was conducted to investigate the clinical characteristics of disturbances in glucose homeostasis and their associations with 90-day mortality in patients with HBV-related ACLF.

Methods Ninety-six patients with HBV-related ACLF without pre-existing diabetes were prospectively included. Glucose abnormalities were diagnosed based on fasting plasma glucose and oral glucose tolerance test results on admission and during follow-up. Homeostasis model assessment was used to establish insulin resistance (HOMA2-IR), insulin sensitivity (HOMA2-IS) and HOMA2- $\beta$-cell function (HOMA2- $\beta$ ). Multivariate Cox proportional hazards analysis was used to identify independent risk factors for death within 90 days after admission.

Results Among 96 patients with ACLF, 51 (53.1\%) had diabetes, 29 (30.2\%) had impaired glucose tolerance (IGT), and 17 $(17.7 \%)$ had hypoglycemia. Patients with diabetes had significantly lower levels of HOMA2- $\beta$ than did patients with normal glucose tolerance. Of 22 patients with diabetes or IGT and without anti-hyperglycemic treatment, 8 (36.4\%) exhibited regression of their glucose metabolism disorders after a follow-up of $32.8 \pm 28.8$ days, and higher platelet levels were associated with regression. Twenty-five patients $(25.0 \%)$ with ACLF died of liver failure within 90 days. Diabetes [odds ratio (OR) $3.601,95 \%$ confidence interval (CI) 1.342-9.661] and age (OR 1.045, 95\% CI 1.010-1.082) were the independent risk factors associated with mortality.
\end{abstract}

Conclusion Impaired pancreatic $\beta$-cell function is related to diabetes development, and diabetes is associated with high mortality in patients with chronic HBV-related ACLF.

Keywords Acute-on-chronic liver failure $\cdot$ Oral glucose tolerance test $\cdot$ Diabetes $\cdot$ Hypoglycemia $\cdot$ Impaired glucose tolerance $\cdot$ Prognosis $\cdot \beta$-Cell function $\cdot$ Hepatogenous diabetes $\cdot$ Stress-induced hyperglycemia $\cdot$ Hepatitis B virus

Han $\mathrm{Hu}$ and Xinxin Hu contributed equally to this work.

Shide Lin

linshide6@zmu.edu.cn

1 Department of Infectious Diseases, Affiliated Hospital of Zunyi Medical University, 201 Dalian Street,

Zunyi 563003, Guizhou, China

2 Department of Epidemiology and Health Statistics, School of Public Health, Zunyi Medical University, Zunyi, Guizhou, China

\section{Introduction}

The liver plays an important role in glucose metabolism. Excessive liver damage disturbs glucose homeostasis, thus resulting in hypoglycemia, impaired glucose tolerance (IGT) and diabetes in patients with severe liver diseases [1]. Diabetes, second to severely impaired liver function, is known as hepatogenous diabetes (HD), although the World Health Organization does not classify it as an independent disease [2]. Hyperglycemia can also occur in patients with severe acute diseases, such as sepsis [3], coronavirus disease 2019[4], and myocardial infarction [5], and is defined as stress-induced hyperglycemia [6]. 
Acute-on-chronic liver failure (ACLF) is a severe liver injury accompanied by reduced liver function, organ failure and systemic inflammation in patients with chronic liver diseases [7]. In China, ACLF occurs most frequently in patients with chronic hepatitis B or hepatitis B virus (HBV)-related cirrhosis. Patients with HBV-related ACLF have different degrees of liver fibrosis and severe acute systemic inflammation [8]. Previous studies found that HD is a common complication in patients with liver cirrhosis. The HD prevalence in patients with liver cirrhosis depends on the degree of liver fibrosis, the etiology of the chronic liver disease and the severity of the liver injury $[9,10]$. Hypoglycemia and temporal hyperglycemia can also occur in patients with severe acute hepatitis and acute liver failure (ALF) [11]. Therefore, glucose metabolism disorders in patients with ACLF may differ from those in patients with liver cirrhosis. In patients with ACLF, glucose metabolism disorders may result from both the loss of liver function and the stress response to acute systemic inflammation. However, the clinical characteristics of glucose metabolism disorders in patients with ACLF remain unclear.

Both hypoglycemia and hyperglycemia have been closely correlated with high mortality in patients with severe diseases [3, 12]. Previous studies have found that diabetes was associated with a poor long-term prognosis and high risk of complications, such as bacterial infections, esophageal variceal bleeding, hepatic encephalopathy, and hepatocellular carcinoma, in patients with liver cirrhosis [13-16]. However, the diagnostic methods used to test for diabetes and the etiologies of the patients varied widely in these studies. Moreover, the effects of diabetes on short-term mortality in patients with liver cirrhosis remain controversial, and the effects of glucose metabolism disorders on the prognosis of patients with ACLF are unknown [17, 18]. Here, we evaluated the clinical characteristics of disturbances in glucose homeostasis and their associations with 90-day mortality in patients with HBV-related ACLF.

\section{Patients and methods}

\section{Study participants}

We prospectively included 206 patients with ACLF associated with chronic HBV infection who were hospitalized in the Department of Infectious Diseases at the Affiliated Hospital of Zunyi Medical University (Zunyi, China) from October 2017 to December 2020. Forty-six patients who did not undergo an oral glucose tolerance test (OGTT) on admission because they were complicated with esophageal or gastric variceal bleeding, hepatic encephalopathy or hepato-renal syndrome were excluded. Fifty-one patients were excluded for coexistence of other liver diseases or pre-existing diabetes. Six patients who were lost to followup within 90 days and 7 with incomplete clinical data were also excluded. Finally, 96 patients were enrolled in the study. The patient screening flow chart is shown in Fig. 1.

\section{Diagnostic criteria for ACLF, liver cirrhosis and bacterial infections}

ACLF was diagnosed as the recent development of jaundice [total bilirubin (TBil) $\geq 5 \times$ upper limits of normal (ULN)] and coagulopathy [prothrombin activity (PTA) $<40 \%$ or international normalized ratio (INR) $\geq 1.5$ ], complicated within 4 weeks by ascites and/or encephalopathy in patients with previously diagnosed or undiagnosed chronic liver disease [19].

Liver cirrhosis was diagnosed based on previous liver biopsy findings, ultrasonography, computed tomography, liver stiffness measurement or magnetic resonance imaging findings. Patients with irregular and nodular livers, small and shrunken livers, splenomegaly and hyper-splenism, or evidence of portosystemic collaterals together with impaired liver synthetic function were diagnosed with liver cirrhosis [20]. Patients with liver cirrhosis were divided into A, B and $\mathrm{C}$ groups based on Child-Pugh scores.

Bacterial infections were diagnosed as per the following criteria [21]: (1) spontaneous bacterial peritonitis: ascitic fluid polymorphonuclear cells $>250 / \mathrm{mL}$ or positive ascitic fluid cultures; (2) bacteremia: positive blood cultures without a source of infection; (3) pneumonia: new pulmonary infiltrate with fever $\left(>38{ }^{\circ} \mathrm{C}\right)$ with any respiratory symptoms (e.g., cough, sputum, dyspnea) or any findings on auscultation (rales or crepitation), or white blood cell (WBC) counts $>10 \times 10^{9} / \mathrm{L}$ or $<4 \times 10^{9} / \mathrm{L}$; and (4) other bacterial infections, including skin infections, intra-abdominal infections, and infections of unknown origin.

\section{Clinical and laboratory assessment}

Patient demographics, clinical and laboratory variables, and imaging findings were collected within $24 \mathrm{~h}$ before and after admission.

Fasting plasma glucose (FPG), fasting insulin (FINS) and fasting C-peptide (FCP) were detected within $24 \mathrm{~h}$ after admission. OGTTs were performed as described by the World Health Organization with an oral anhydroglucose load of $75 \mathrm{~g}$, and glycemia was measured at 0 and $2 \mathrm{~h}$. Glucose abnormalities were diagnosed based on the American Diabetes Association criteria [22]. Hypoglycemia was defined as FPG $<3.8 \mathrm{mmol} / \mathrm{L}$; diabetes was established as FPG $\geq 7 \mathrm{mmol} / \mathrm{L}$ or OGTT 2-h plasma glucose (OGTT $2 \mathrm{~h}-\mathrm{PG}) \geq 11.1 \mathrm{mmol} / \mathrm{L}$. IGT included patients with impaired fasting glucose (FPG of $\geq 5.6 \mathrm{mmol} / \mathrm{L}$ but $<7 \mathrm{mmol} / \mathrm{L}$ ) and/or impaired glucose tolerance (OGTT 
Fig. 1 The patient screening flow chart. ACLF, acute-onchronic liver failure; HBV, hepatitis B virus; OGTT, oral glucose tolerance test

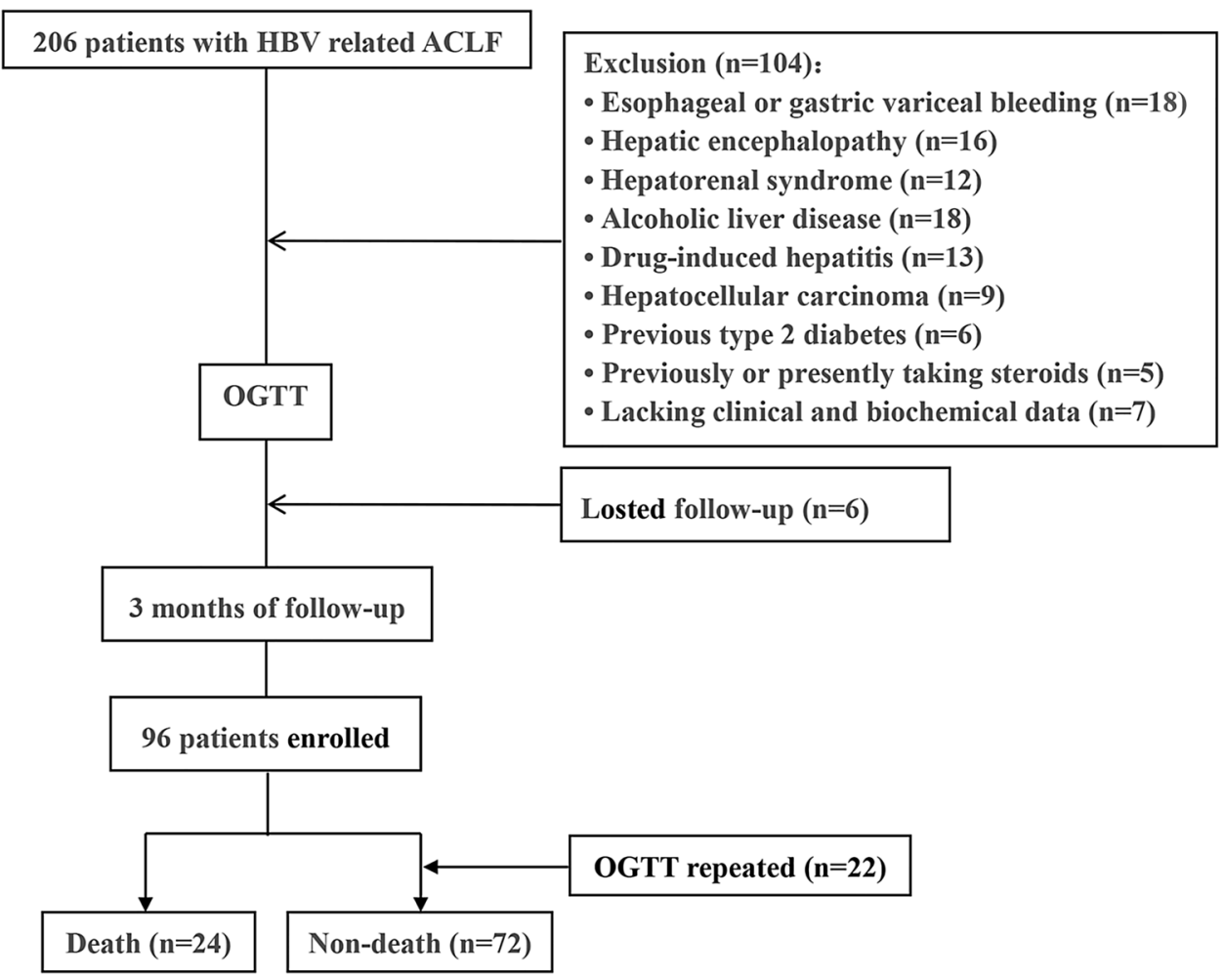

$2 \mathrm{~h}-\mathrm{PG}$ of $\geq 7.8 \mathrm{mmol} / \mathrm{L}$ and $<11.1 \mathrm{mmol} / \mathrm{L})$. Because glycated hemoglobin (HbA1c) levels may be inappropriately normal in patients with severe liver cirrhosis owing to reduced erythrocyte lifespans [2], we excluded $\mathrm{HbA} 1 \mathrm{c}$ from this study. The homeostasis model of assessment 2-insulin resistance (HOMA2-IR), HOMA2-insulin sensitivity (HOMA2-IS), and HOMA2- $\beta$-cell function (HOMA2- $\beta$ ) indices were estimated with the HOMA2 calculator, version 2.2 released by the Diabetes Trials Unit at the Oxford Centre for Diabetes, Endocrinology and Metabolism, University of Oxford [23]. Liver disease severity was assessed using the model for end-stage liver disease (MELD) score, which was calculated using the following formula: MELD score $=3.78 \times \ln [\mathrm{TBil}(\mathrm{mg} / \mathrm{dL})]+11.2 \times \ln [\mathrm{INR}]+9.57 \times \ln$ $[\mathrm{Cr}(\mathrm{mg} / \mathrm{dL})]+6.43 \times($ constant for liver disease etiology $=0$ if cholestatic or alcoholic, otherwise $=1)$.

\section{Therapeutic method and follow-up}

After admission, all patients received intensive supportive treatment, such as liver protection, albumin supplementation, maintenance of water and electrolyte balance, plasma transfusion. 78 patients received plasma exchange. All patients received antiviral therapy (e.g., tenofovir, entecavir, lamivudine, ) within 3 days after admission.

For patients with diabetes, oral hypoglycemic agents or insulin were initiated if dietary therapy was insufficient to obtain good glycemic control. For patients with IGT, no specific treatment was administered. All patients were followed for up to 90 days after admission. In 22 patients with diabetes or IGT without oral hypoglycemic agents or insulin treatment, an OGTT was repeated to evaluate the regression of their glucose metabolism disorders. Based on their OGTT results at baseline and follow-up, patients were retrospectively assigned to one of two outcomes groups: (1) regression: from diabetes or IGT to normal glucose tolerance (NGT) or from diabetes to IGT or (2) non-regression: no change in their glucose metabolism disorders or progression from IGT or NGT to diabetes or from NGT to IGT.

\section{Statistical analysis}

SPSS, version 19.0 (IBM Corp., Armonk, NY, USA) was used for statistical processing. Differences between groups were assessed using unpaired t tests, paired t tests, Mann-Whitney $U$ tests, one-way analysis of variance (ANOVA), or Wilcoxon rank-sum test, followed by the Bonferroni test for post hoc multiple comparisons. The Chi-square test was used for categorical data. Univariate logistic regression was used to determine the risk factors associated with regression of glucose metabolism disorders. A multivariate Cox proportional hazards model was used to determine independent predictive factors of mortality. Cumulative survival was analyzed using the Kaplan-Meier method, and the curves were compared using the log-rank test. $p<0.05$ was considered statistically significant. 


\section{Results}

\section{Disturbances in glucose homeostasis in patients with ACLF}

Of 96 patients with ACLF, 51 (53.1\%) had diabetes, 29 (30.2\%) had IGT, and 17 (17.7\%) had hypoglycemia. Of the 51 patients with diabetes, 4 had only high FPG, 40 had only high OGTT 2 h-PG, and 7 had both high FPG and high OGTT 2 h-PG. Of the 17 patients with hypoglycemia, 4 had NGT, 6 had IGT, and 7 had diabetes. Based on the OGTT assay, 13/17 patients (76.5\%) with fasting hypoglycemia had IGT or diabetes.

Patients with diabetes had significantly lower HOMA2- $\beta$ levels than did patients with NGT (Table 1). No other clinical or laboratory parameters differed significantly among patients with NGT, IGT and diabetes. Patients with hypoglycemia had significantly higher HOMA2-IS, HOMA2- $\beta$, alanine aminotransferase, aspartate aminotransferase, and TBil levels and MELD scores and significantly lower HOMA2-IR, FINS and FCP levels than did patients without hypoglycemia (Supplementary Table 1).

\section{Dynamic changes in glucose homeostasis in patients with ACLF}

In 22 patients ( 8 with IGT and 14 with diabetes) who received no anti-hyperglycemic treatment, an OGTT was repeated to evaluate the regression of their glucose metabolism disorders after a median of $32.8 \pm 28.8$ days. Most patients exhibited improved liver function when the OGTT was repeated (Supplementary Table 2). Eight patients had regression of their glucose metabolism disorders ( 2 with diabetes and 1 with IGT regressed to NGT, 5 with diabetes regressed to IGT). Fourteen patients did not regress (one progressed from IGT to diabetes; the others remained unchanged; Fig. 2). Patients whose glucose metabolism disorders regressed had significantly higher platelets (PLTs) and albumin (ALB) levels and a significantly lower prevalence of Child-Pugh class $\mathrm{C}$ liver cirrhosis than did patients without regression when the OGTT was repeated (Table 2). Univariate and multivariate logistic analysis showed that PLT and ALB levels were the factors associated with regressed glucose metabolism disorders. PLTs [odds ratio (OR) 1.025, 95\% confidence interval (CI) 1.001-1.050] were independently associated with regressed glucose metabolism disorders in patients with ACLF.

\section{Diabetes was associated with high mortality in patients with ACLF}

Twenty-two patients died from liver failure and two patients died from esophageal or gastric variceal bleeding within
90 days after admission; 19 had diabetes, and 5 had NGT. Among the patients who died, 6 (25\%) had hypoglycemia; this number did not significantly differ from that of surviving patients $(15.3 \%, p=0.355)$. The proportional of patients who received the treatment of plasma exchange did not significantly differ between the dead and surviving patients (Table 3).

Patients who died within 90 days were significantly older and had a higher prevalence of diabetes, higher INR and TBil levels, higher MELD scores, a lower IGT prevalence and lower FINS levels than did surviving patients (Fig. 3). Univariate analysis showed that age, diabetes, INR, TBil and MELD score were the risk factors associated with death (Table 4). Because the MELD score was calculated from the INR and TBil, we selected age, diabetes and MELD score for multivariate analysis. Age (OR 1.045, 95\% CI 1.010-1.082) and diabetes (OR 3.601, 95\% CI 1.342-9.661) were the independent risk factors associated with death in patients with chronic HBV-related ACLF. Patients with both chronic HBV-related ACLF and diabetes had significantly higher mortality than did those with only HBV-related ACLF (Fig. 4).

\section{Discussion}

In patients with ACLF, acute and chronic liver dysfunction may result in HD, and severe liver and systemic inflammation may result in stress-induced hyperglycemia. Hypoglycemia occurs frequently in both patients with HD and patients with acute stress. Clinically, HD is difficult to distinguish from stress-induced hyperglycemia, and HD-related hypoglycemia is difficult to distinguish from hypoglycemia induced by acute stress. In this study, we excluded patients with pre-existing diabetes. Glucose metabolism disorders in the patients in this study were mostly due to severe liver injury and severe systemic inflammation. Most patients with diabetes had normal FPG, and most patients with hypoglycemia had diabetes or IGT, demonstrating that complex disturbances in glucose homeostasis existed in patients with ACLF.

Previous studies have found that both pancreatic $\beta$-cell dysfunction and increased insulin resistance play central roles in HD development in patients with liver cirrhosis [24]. Stress-induced hyperglycemia is also considered a result of pancreatic failure in compensating for increased insulin production and release [25]. In the current study, patients with diabetes had significantly lower HOMA2- $\beta$ levels than did patients with NGT, we did not find the associations of glucose abnormalities with C-reactive protein, neutrophilto-lymphocyte ratio and white blood count, demonstrating that an impaired pancreatic $\beta$-cell response to acute liver 
Table 1 Clinical characteristics and disturbances in glucose homeostasis in patients with acute-on-chronic liver failure

\begin{tabular}{|c|c|c|c|c|}
\hline Variables & NGT $(n=16)$ & IGT $(n=29)$ & Diabetes $(n=51)$ & $p$ value \\
\hline $\operatorname{Men}(n \%)$ & $11(68.8)$ & $25(86.2)$ & $43(84.3)$ & 0.292 \\
\hline Age (years) & $50.4 \pm 15.7$ & $44.1 \pm 13.2$ & $49.3 \pm 9.8$ & 0.201 \\
\hline BMI $\left(\mathrm{kg} / \mathrm{m}^{2}\right)$ & $22.9 \pm 3.5$ & $23.3 \pm 2.4$ & $23.3 \pm 2.8$ & 0.885 \\
\hline Child-Pugh ( $n \%)$ & & & & 0.548 \\
\hline A & $0(0.0)$ & $0(0.0)$ & $0(0.0)$ & \\
\hline B & $2(12.5)$ & $3(10.3)$ & $3(5.9)$ & \\
\hline $\mathrm{C}$ & $14(87.5)$ & $24(82.8)$ & $47(92.2)$ & \\
\hline HOMA2-IR & $2.4 \pm 1.7$ & $2.6 \pm 1.3$ & $2.9 \pm 2.3$ & 0.677 \\
\hline HOMA2-IS & $43.9(31.7,97.8)$ & $45.6(31.1,55.6)$ & $48.8(27.0,73.1)$ & 0.760 \\
\hline HOMA2- $\beta(\%)$ & $251.6(170.7,310.5)$ & $197.2(161.0,258.1)$ & $162.2(119.9,228.7)^{\#}$ & 0.045 \\
\hline FINS $(\mu \mathrm{IU} / \mathrm{mL})$ & $7.3(2.9,18.3)$ & $10.9(5.5,20.5)$ & $9.2(5.9,17.4)$ & 0.642 \\
\hline FCP (pmol/L) & $1120.0(519.7,1558.8)$ & $1017.0(844.0,1244.0)$ & $941.9(631.1,1499.0)$ & 0.792 \\
\hline INR & $1.82 \pm 0.38$ & $1.87 \pm 0.35$ & $1.97 \pm 0.47$ & 0.328 \\
\hline PTA $(\%)$ & $34.9 \pm 9.0$ & $35.6 \pm 8.8$ & $35.2 \pm 7.5$ & 0.962 \\
\hline ALT (U/L) & $127.0(31.5,227.5)$ & $139.0(32.5,420.5)$ & $191.0(60.0,574.0)$ & 0.223 \\
\hline AST (U/L) & $119.0(75.0,235.3)$ & $169.0(61.5,349.0)$ & $190.0(109.0,541.0)$ & 0.240 \\
\hline TBil $(\mu \mathrm{mol} / \mathrm{L})$ & $242.4(136.1,389.0)$ & $237.6(121.0,324.3)$ & $319.4(166.6,405.0)$ & 0.174 \\
\hline $\operatorname{ALB}(g / L)$ & $28.5 \pm 5.5$ & $28.0 \pm 4.2$ & $30.2 \pm 5.7$ & 0.178 \\
\hline $\mathrm{Na}^{+}(\mathrm{mmol} / \mathrm{L})$ & $136.07 \pm 3.90$ & $135.03 \pm 2.97$ & $135.20 \pm 4.08$ & 0.654 \\
\hline $\mathrm{Cr}(\mu \mathrm{mol} / \mathrm{L})$ & $77.0(53.5,118.8)$ & $75.0(64.0,86.5)$ & $74.0(64.0,81.0)$ & 0.819 \\
\hline $\mathrm{UA}(\mu \mathrm{mol} / \mathrm{L})$ & $198.5(127.3,306.3)$ & $209.0(163.0,321.0)$ & $171.0(129.0,229.0)$ & 0.060 \\
\hline $\mathrm{TG}(\mathrm{mmol} / \mathrm{L})$ & $1.2 \pm 0.6$ & $1.4 \pm 0.7$ & $1.4 \pm 0.6$ & 0.511 \\
\hline $\mathrm{TC}(\mathrm{mmol} / \mathrm{L})$ & $2.5 \pm 1.1$ & $2.7 \pm 1.0$ & $2.8 \pm 1.4$ & 0.696 \\
\hline HDL (mmol/L) & $0.40 \pm 0.20$ & $0.56 \pm 0.33$ & $0.56 \pm 0.40$ & 0.269 \\
\hline LDL (mmol/L) & $1.8 \pm 0.7$ & $1.8 \pm 0.6$ & $1.8 \pm 0.9$ & 0.955 \\
\hline WBC $\left(10^{9} / \mathrm{L}\right)$ & $5.7 \pm 2.9$ & $6.0 \pm 3.0$ & $7.0 \pm 3.4$ & 0.249 \\
\hline NLR $(\%)$ & $3.7(3.1,5.5)$ & $3.3(2.4,5.8)$ & $3.4(2.0,7.1)$ & 0.821 \\
\hline $\operatorname{PLT}\left(10^{9} / \mathrm{L}\right)$ & $67.0(59.3,72.5)$ & $94.0(44.5,159.0)$ & $97.0(56.0,122.0)$ & 0.163 \\
\hline CRP (mg/L) & $8.9(4.3,21.1)$ & $9.1(5.6,14.2)$ & $12.2(5.2,18.0)$ & 0.533 \\
\hline lgHBV-DNA (copies/mL) & $4.2 \pm 2.7$ & $5.0 \pm 2.1$ & $5.1 \pm 2.3$ & 0.374 \\
\hline AFP (ng/mL) & $35.9(3.3,133.6)$ & $36.8(10.4,104.5)$ & $34.3(16.8,113.8)$ & 0.906 \\
\hline MELD score & $22.0 \pm 6.2$ & $20.7 \pm 4.5$ & $22.2 \pm 5.3$ & 0.455 \\
\hline Death $(n \%)$ & $5(31.3)$ & $0(0.0)$ & $19(37.3)$ & 0.001 \\
\hline Infection $(n \%)$ & $10(62.5)$ & $17(58.6)$ & $32(62.7)$ & 0.932 \\
\hline
\end{tabular}

AFP, alpha fetoprotein; ALB, albumin; ALT, alanine aminotransferase; AST, aspartate aminotransferase; BMI, body mass index; Cr, creatinine; CRP, C-reactive protein; FCP, fasting C-peptide; FINS, fasting insulin; HBV, hepatitis B virus; HBV-DNA, hepatitis B virus-DNA; HDL, high-density lipoprotein; HOMA2-IR, homeostasis model assessment 2-insulin resistance; HOMA2-IS, homeostasis model assessment 2-insulin sensitivity; HOMA2- $\beta$, homeostasis model assessment 2 - $\beta$-cell function; IGT, impaired glucose tolerance; INR, international normalized ratio; LDL, low-density lipoprotein; MELD, model for end-stage liver disease; NLR, neutrophil-to-lymphocyte ratio; $\mathrm{Na}^{+}$, sodium; NGT, normal glucose tolerance; PLT, platelet; PTA, prothrombin activity; TBil, total bilirubin; TC, total cholesterol; TG, triglyceride; UA, uric acid; WBC, white blood cell

${ }^{\#}$ Compared with the NGT group, $p<0.05$. Data are presented as the mean $\pm \mathrm{SD}, n(\%)$, or median (interquartile range). Differences between groups were assessed using one-way ANOVA or chi-square tests

inflammation and damage plays a determining role in the development of diabetes in patients with ACLF.

In patients with HD or stress-induced hyperglycemia, managing the hyperglycemia remains a clinical challenge. Hyperglycemia increased the mortality rates of patients with liver cirrhosis and critically ill patients. However, strictly controlling patients' hyperglycemia increased the prevalence of hypoglycemia, which was also associated with high mortality [26]. Most patients with ACLF have severe digestive symptoms, making it impossible to perform the OGTT, and managing diabetes in patients with ACLF is especially difficult. Therefore, the dynamic changes in glucose metabolism 


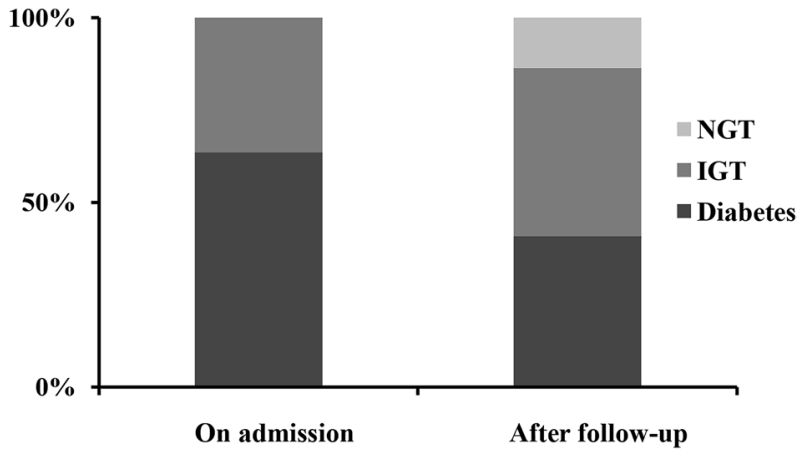

Fig. 2 Dynamic changes in glucose homeostasis in patients with hepatitis B virus-related acute-on-chronic liver failure. NGT, normal glucose tolerance; IGT, impaired glucose tolerance

disorders in patients with ACLF must be better understood. In the current study, eight (36.4\%) of 22 patients with ACLF exhibited regressed glucose metabolism disorders and improved liver function during the $32.8 \pm 28.8$-day followup. PLT levels were positively associated with the regression. Our results indicated that in patients with ACLF and sustained low PLTs, OGTTs should be repeated to evaluate the regression of the glucose metabolism disorders.

One major finding in this study was that diabetes was an independent risk factor for 90-day mortality in patients with ACLF. Previous studies showed that hyperglycemia accelerated the progression of liver fibrosis and provoked liver inflammation [27]. Several clinical studies demonstrated that HD or pre-existing diabetes carried high risks for poor long-term outcomes and complications in patients with liver cirrhosis $[16,28]$. One study evaluated the association of $\mathrm{HD}$ and 30-day survival in 78 patients with decompensated liver cirrhosis and found that HD and IGT better predicted a poor 30-day survival than did NGT [18]. Another study evaluated the association between type 2 diabetic mellitus (T2DM) and the prognosis of patients with ACLF, they found 38/222 (17.1\%) patients had T2DM and T2DM was

Table 2 The clinical characteristics and disturbances in glucose homeostasis in patients with acute-on-chronic liver failure during follow-up

\begin{tabular}{|c|c|c|c|c|}
\hline Variables & Total $(n=22)$ & Without regression $(n=14)$ & With regression $(n=8)$ & $p$ value \\
\hline $\operatorname{Men}(n \%)$ & $18(81.8)$ & $11(78.6)$ & $7(87.5)$ & $1.000^{\mathrm{a}}$ \\
\hline Age (years) & $47.4 \pm 10.6$ & $47.4 \pm 10.2$ & $47.3 \pm 12.1$ & $0.971^{\mathrm{b}}$ \\
\hline BMI $\left(\mathrm{kg} / \mathrm{m}^{2}\right)$ & $23.8 \pm 1.8$ & $24.2 \pm 1.47$ & $23.1 \pm 2.2$ & $0.171^{\mathrm{b}}$ \\
\hline Child-Pugh ( $n \%)$ & & & & $0.038^{\mathrm{a}}$ \\
\hline A & $2(9.1)$ & $0(0.0)$ & $2(25.0)$ & \\
\hline B & $10(45.5)$ & $6(42.9)$ & $4(50.0)$ & \\
\hline $\mathrm{C}$ & $9(40.9)$ & $8(57.1)$ & $1(12.5)$ & \\
\hline HOMA2-IR & $2.7 \pm 1.6$ & $2.7 \pm 1.7$ & $2.7 \pm 1.3$ & $0.988^{\mathrm{b}}$ \\
\hline HOMA2-IS & $47.1 \pm 25.0$ & $45.6 \pm 18.3$ & $49.6 \pm 35.3$ & $0.728^{\mathrm{b}}$ \\
\hline HOMA2- $\beta(\%)$ & $171.5 \pm 90.1$ & $148.9 \pm 78.3$ & $211.2 \pm 100.7$ & $0.121^{\mathrm{b}}$ \\
\hline FINS $(\mu \mathrm{IU} / \mathrm{mL})$ & $18.5 \pm 11.7$ & $14.6 \pm 6.8$ & $25.3 \pm 15.6$ & $0.101^{\mathrm{b}}$ \\
\hline $\mathrm{FCP}(\mathrm{pmol} / \mathrm{L})$ & $1252.7 \pm 846.9$ & $1253.1 \pm 989.6$ & $1252.2 \pm 577.3$ & $0.998^{\mathrm{b}}$ \\
\hline INR & $1.55 \pm 0.45$ & $1.65 \pm 0.46$ & $1.37 \pm 0.38$ & $0.155^{\mathrm{b}}$ \\
\hline PTA (\%) & $47.8(39.9,71.6)$ & $47.0(34.6,71.6)$ & $56.2(41.6,85.6)$ & $0.322^{\mathrm{c}}$ \\
\hline ALT (U/L) & $38.0(22.0,61.3)$ & $32.5(21.3,55.0)$ & $52.0(34.5,112.5)$ & $0.108^{\mathrm{c}}$ \\
\hline AST (U/L) & $66.2 \pm 35.5$ & $63.0 \pm 31.1$ & $71.7 \pm 44.1$ & $0.591^{\mathrm{b}}$ \\
\hline TBil $(\mu \mathrm{mol} / \mathrm{L})$ & $69.9(44.7,155.8)$ & $69.0(55.1,275.4)$ & $56.9(26.4,138.3)$ & $0.219^{\mathrm{c}}$ \\
\hline ALB (g/L) & $32.1 \pm 3.3$ & $31.0 \pm 2.7$ & $34.1 \pm 3.5$ & $0.032^{\mathrm{b}}$ \\
\hline WBC $\left(10^{9} / \mathrm{L}\right)$ & $4.74 \pm 2.62$ & $4.82 \pm 2.99$ & $4.62 \pm 2.02$ & $0.867^{\mathrm{b}}$ \\
\hline PLTs $\left(10^{9} / \mathrm{L}\right)$ & $74.0(39.2,102.0)$ & $45.5(36.0,88.5)$ & $99.0(78.2,184.7)$ & $0.041^{\mathrm{c}}$ \\
\hline lgHBV-DNA (copies/mL) & $1.48(1.48,3.01)$ & $1.48(1.33,2.84)$ & $1.99(1.48,4.07)$ & $0.152^{\mathrm{c}}$ \\
\hline
\end{tabular}

Data are presented as the mean $\pm \mathrm{SD}, n(\%)$, or median (interquartile range)

ALB, albumin; ALT, alanine aminotransferase; AST, aspartate aminotransferase; BMI, body mass index; FCP, fasting C-peptide; FINS, fasting insulin; HBV-DNA, hepatitis B virus-DNA; HD, hepatogenous diabetes; HOMA2-IR, homeostasis model assessment 2-insulin resistance; HOMA2-IS, homeostasis model assessment 2-insulin sensitivity; HOMA2- $\beta$, homeostasis model assessment 2 - $\beta$-cell function; IGT, impaired glucose tolerance; INR, international normalized ratio; NGT, normal glucose tolerance; PLTs, platelets; PTA, prothrombin activity; TBil, total bilirubin; WBC, white blood cells

${ }^{\mathrm{a} C h i}$-square test results

${ }^{\mathrm{b}} t$ test results

${ }^{\mathrm{c}}$ Wilcoxon rank-sum test 
Table 3 Clinical characteristics and disturbances in glucose homeostasis in dead and surviving patients with acute-on-chronic liver failure

\begin{tabular}{|c|c|c|c|c|}
\hline Variables & Total $(n=96)$ & Death $(n=24)$ & Non-death $(n=72)$ & $p$ value \\
\hline $\operatorname{Men}(n \%)$ & $79(82.3)$ & $20(83.3)$ & $59(81.9)$ & $1.000^{\mathrm{a}}$ \\
\hline Age (years) & $47.9 \pm 12.1$ & $53.4 \pm 10.9$ & $46.1 \pm 12.0$ & $0.010^{\mathrm{b}}$ \\
\hline BMI $\left(\mathrm{kg} / \mathrm{m}^{2}\right)$ & $23.2 \pm 2.8$ & $22.8 \pm 2.4$ & $23.4 \pm 2.9$ & $0.429^{\mathrm{b}}$ \\
\hline Hypoglycemia ( $n \%$ ) & $17(17.7)$ & $6(25.0)$ & $11(15.3)$ & $0.355^{\mathrm{a}}$ \\
\hline IGT $(n \%)$ & $29(30.2)$ & $0(0.0)$ & $29(40.3)$ & $0.001^{\mathrm{a}}$ \\
\hline Diabetes $(n \%)$ & $51(53.1)$ & $19(79.2)$ & $32(44.4)$ & $0.001^{\mathrm{a}}$ \\
\hline Child-Pugh (n\%) & & & & $0.608^{\mathrm{a}}$ \\
\hline A & $0(0.0)$ & $0(0.0)$ & $0(0.0)$ & \\
\hline $\mathrm{B}$ & $8(8.3)$ & $1(4.2)$ & $7(9.7)$ & \\
\hline $\mathrm{C}$ & $85(88.5)$ & $23(95.8)$ & $62(86.1)$ & \\
\hline HOMA2-IR & $2.2(1.4,3.3)$ & $1.5(1.0,3.7)$ & $2.2(1.7,3.3)$ & $0.100^{\mathrm{c}}$ \\
\hline HOMA2-IS & $47.1(30.1,73.7)$ & $66.8(27.0,94.7)$ & $45.0(30.5,59.8)$ & $0.104^{\mathrm{c}}$ \\
\hline HOMA2- $\beta(\%)$ & $186.1(137.4,258.4)$ & $165.6(104.6,255.0)$ & $188.8(147.0,269.9)$ & $0.288^{\mathrm{c}}$ \\
\hline FPG (mmol/L) & $5.3 \pm 2.8$ & $5.8 \pm 3.9$ & $5.2 \pm 2.3$ & $0.468^{\mathrm{b}}$ \\
\hline OGTT 2 h-PG (mmol/L) & $11.3 \pm 4.5$ & $12.1 \pm 5.4$ & $11.0 \pm 4.2$ & $0.290^{\mathrm{b}}$ \\
\hline FINS $(\mu \mathrm{IU} / \mathrm{mL})$ & $9.7(5.5,18.3)$ & $7.7(3.2,9.6)$ & $11.7(5.9,20.6)$ & $0.011^{\mathrm{c}}$ \\
\hline $\mathrm{FCP}(\mathrm{pmol} / \mathrm{L})$ & $993.3(633.3,1492.5)$ & $727.4(520.7,1285.0)$ & $1018.5(681.7,1524.5)$ & $0.072^{\mathrm{c}}$ \\
\hline INR & $1.92 \pm 0.42$ & $2.10 \pm 0.53$ & $1.86 \pm 0.37$ & $0.048^{\mathrm{b}}$ \\
\hline PTA (\%) & $35.3 \pm 8.1$ & $33.6 \pm 7.2$ & $35.8 \pm 8.3$ & $0.247^{b}$ \\
\hline ALT (U/L) & $139.0(42.8,423.3)$ & $314.5(70.0,521.8)$ & $125.0(40.3,410.8)$ & $0.390^{\mathrm{c}}$ \\
\hline AST (U/L) & $155.5(83.5,380.8)$ & $178.0(102.0,528.8)$ & $155.5(79.3,361.8)$ & $0.397^{\mathrm{c}}$ \\
\hline TBil $(\mu \mathrm{mol} / \mathrm{L})$ & $250.1(150.3,373.3)$ & $399.6(232.7,467.2)$ & $222.8(131.6,347.5)$ & $0.000^{\mathrm{c}}$ \\
\hline $\operatorname{ALB}(\mathrm{g} / \mathrm{L})$ & $29.2 \pm 5.3$ & $30.6 \pm 7.1$ & $28.8 \pm 4.5$ & $0.139^{\mathrm{b}}$ \\
\hline $\mathrm{Na}^{+}(\mathrm{mmol} / \mathrm{L})$ & $135.3 \pm 3.7$ & $135.4 \pm 4.6$ & $135.3 \pm 3.4$ & $0.918^{\mathrm{b}}$ \\
\hline $\mathrm{Cr}(\mu \mathrm{mol} / \mathrm{L})$ & $80.1 \pm 34.8$ & $79.1 \pm 38.1$ & $80.5 \pm 33.9$ & $0.871^{\mathrm{b}}$ \\
\hline $\mathrm{UA}(\mu \mathrm{mol} / \mathrm{L})$ & $211.9 \pm 102.6$ & $197.2 \pm 121.7$ & $216.9 \pm 95.9$ & $0.418^{\mathrm{b}}$ \\
\hline $\mathrm{TG}(\mathrm{mmol} / \mathrm{L})$ & $1.4 \pm 0.6$ & $1.4 \pm 0.5$ & $1.4 \pm 0.6$ & $0.944^{\mathrm{b}}$ \\
\hline $\mathrm{TC}(\mathrm{mmol} / \mathrm{L})$ & $2.7 \pm 1.2$ & $2.9 \pm 1.6$ & $2.6 \pm 1.0$ & $0.376^{\mathrm{b}}$ \\
\hline HDL (mmol/L) & $0.53 \pm 0.35$ & $0.50 \pm 0.40$ & $0.54 \pm 0.34$ & $0.606^{\mathrm{b}}$ \\
\hline $\mathrm{LDL}(\mathrm{mmol} / \mathrm{L})$ & $1.8 \pm 0.8$ & $1.9 \pm 1.1$ & $1.8 \pm 0.6$ & $0.500^{\mathrm{b}}$ \\
\hline WBC $\left(10^{9} / \mathrm{L}\right)$ & $6.4 \pm 3.2$ & $7.2 \pm 3.8$ & $6.2 \pm 3.0$ & $0.217^{\mathrm{b}}$ \\
\hline $\operatorname{NLR}(\%)$ & $3.6(2.4,6.0)$ & $4.0(2.9,7.7)$ & $3.4(2.3,5.8)$ & $0.210^{\mathrm{c}}$ \\
\hline PLTs $\left(10^{9} / \mathrm{L}\right)$ & $95.3 \pm 51.3$ & $101.5 \pm 46.4$ & $93.2 \pm 53.0$ & $0.494^{\mathrm{b}}$ \\
\hline lgHBV-DNA (copies/mL) & $4.9 \pm 2.3$ & $5.2 \pm 2.5$ & $4.8 \pm 2.2$ & $0.460^{\mathrm{b}}$ \\
\hline $\mathrm{CRP}(\mathrm{mg} / \mathrm{L})$ & $9.5(5.3,17.4)$ & $12.7(5.9,17.7)$ & $9.3(5.1,17.4)$ & $0.462^{\mathrm{c}}$ \\
\hline $\mathrm{AFP}(\mathrm{ng} / \mathrm{mL})$ & $34.7(11.4,116.9)$ & $45.8(11.8,149.8)$ & $34.3(11.1,92.8)$ & $0.318^{\mathrm{c}}$ \\
\hline MELD score & $21.7 \pm 5.2$ & $23.7 \pm 5.1$ & $21.0 \pm 5.2$ & $0.025^{\mathrm{b}}$ \\
\hline Infection $(n \%)$ & $59(61.5)$ & $16(66.7)$ & $43(59.7)$ & $0.545^{\mathrm{a}}$ \\
\hline $\mathrm{PE}(n \%)$ & $78(81.3)$ & $21(87.5)$ & $57(79.2)$ & $0.548^{\mathrm{a}}$ \\
\hline
\end{tabular}

Data are presented as the mean $\pm \mathrm{SD}, n(\%)$, or median (interquartile range)

AFP, alpha fetoprotein; ALB, albumin; ALT, alanine aminotransferase; AST, aspartate aminotransferase; BMI, body mass index; Cr, creatinine; CRP, C-reactive protein; FCP, fasting C-peptide; FPG, fasting plasma glucose; FINS, fasting insulin; HBV-DNA, hepatitis B virus-DNA; HDL, high-density lipoprotein; HOMA2-IR, homeostasis model assessment 2-insulin resistance; HOMA2-IS, homeostasis model assessment 2-insulin secretion; HOMA2- $\beta$, homeostasis model assessment $2-\beta$-cell function; INR, international normalized ratio; LDL, low-density lipoprotein; MELD, model for end-stage liver disease; NLR, neutrophil-to-lymphocyte ratio; $\mathrm{Na}^{+}$, sodium; OGTT2h-PG, oral glucose tolerance test $2 \mathrm{~h}$ plasma glucose; PE, plasma exchange; PLTs, platelets; PTA, prothrombin activity; TBil, total bilirubin; TC, total cholesterol; TG, triglyceride; UA, uric acid; WBC, white blood cells

${ }^{\mathrm{a}} \mathrm{Chi}$-square test results

${ }^{\mathrm{b}} t$ test results

${ }^{\mathrm{c}}$ Mann-Whitney $U$ test results 


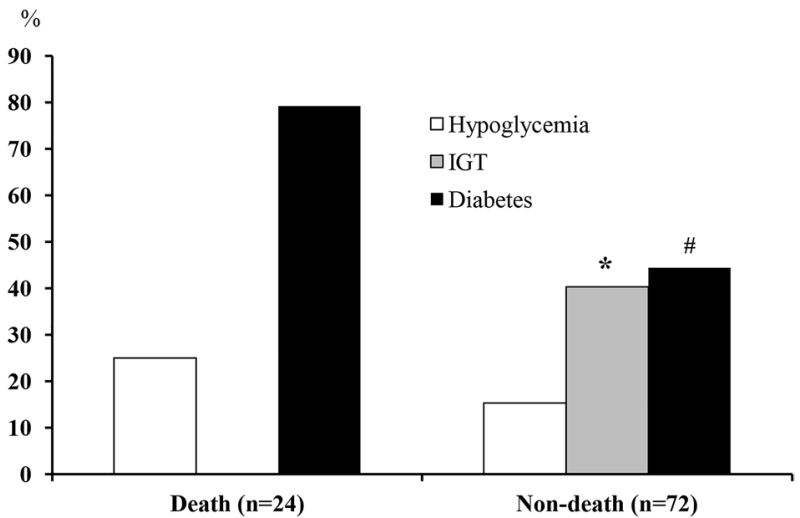

Fig. 3 The clinical characteristics and disturbances in glucose homeostasis of dead and surviving patients with hepatitis B virus-related acute-on-chronic liver failure. IGT, impaired glucose tolerance. ${ }^{*} p<0.01$ versus the group of IGT in death; ${ }^{\#} p<0.01$ versus the group of diabetes in death

associated with poor prognosis in patients with ACLF [29]. However, recently Duseja et al. did not find the association of T2DM with the prognosis in patients with alcohol-associated ACLF [30]. Bianchi et al. also found no association between diabetes and short-term mortality in patients with liver cirrhosis [17]. The reason for the conflicting results in these studies is unknown. Most of above studies did not exclude pre-existing diabetes or perform OGTTs in their study [17, 29, 30]; therefore, the diabetes prevalence might have been underestimated. In this study, we excluded preexisting diabetes and performed OGTTs in 96 patients with ACLF. We found that diabetes resulted from severe liver injury and liver inflammation as one independent risk factor of 90-day mortality in patients with ACLF.

Hypoglycemia was not a risk factor for 90-day mortality in this study. Patients with ALF are generally considered to be at high risk for hypoglycemia [10, 31]; however, to our knowledge, the correlation between hypoglycemia and mortality in patients with ACLF remains unknown. One retrospective study found that hypoglycemia was a risk factor for poor prognosis in patients with decompensated liver cirrhosis [32]; however, no OGTT was performed in that study.
Because the diagnosis of HD in patients with chronic liver diseases depends on OGTT results, and patients with HD are prone to hypoglycemia. Whether the effects of hypoglycemia found in that study actually resulted from the effects of HD requires further analysis. In the current study, the prevalence of hypoglycemia was much lower than that of diabetes, and most patients with hypoglycemia had diabetes. Diabetes was only the risk factor associated with death in patients with ACLF, indicating that diabetes had a worse effect on patients' prognosis than did hypoglycemia in patients with ACLF.

It is difficult to explain why only age and diabetes but not MELD scores were prognostic predictors for patients with ACLF. One explanation was that glucose homeostasis was easily to be disturbed in patients with ACLF, therefore, hyperglycemia predicted the outcomes of patients with ACLF more precisely than MELD. Indeed, previous studies showed that the prognostic value of MELD was

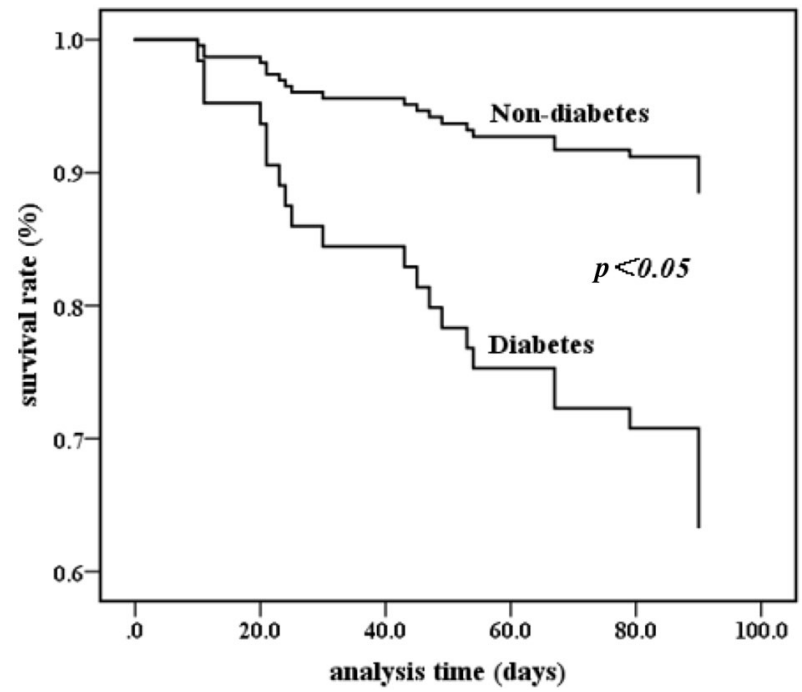

Fig. 4 Cumulative survival rates of patients with hepatitis B virusrelated acute-on-chronic liver failure with and without diabetes
Table 4 Univariate and multivariate analysis of risk factors associated with death in patients with acute-on-chronic liver failure

\begin{tabular}{|c|c|c|c|c|c|c|c|c|}
\hline \multirow[t]{2}{*}{ Variables } & \multicolumn{4}{|c|}{ Univariable analysis } & \multicolumn{4}{|c|}{ Multivariable analysis } \\
\hline & $\beta$ & OR & $95 \% \mathrm{CI}$ & $p$ & $\beta$ & OR & $95 \% \mathrm{CI}$ & $p$ \\
\hline Age & 0.042 & 1.043 & $1.011-1.076$ & 0.009 & 0.044 & 1.045 & $1.010-1.082$ & 0.011 \\
\hline Diabetes & 1.322 & 3.750 & $1.400-10.048$ & 0.009 & 1.281 & 3.601 & $1.342-9.661$ & 0.011 \\
\hline INR & 1.162 & 3.198 & $1.295-7.897$ & 0.012 & & & & \\
\hline TBil & 0.005 & 1.005 & $1.002-1.008$ & 0.000 & & & & \\
\hline MELD score & 0.081 & 1.084 & $1.012-1.162$ & 0.022 & & & & \\
\hline
\end{tabular}

$p<0.05$ was statistically significant

INR, international normalized ratio; MELD, model for end-stage liver disease; TBil, total bilirubin 
not satisfying $[7,19]$. Another explanation was that in this study we had excluded 46 patients with esophageal or gastric variceal bleeding, hepatic encephalopathy or hepatorenal syndrome on admission, for they could not undergo an OGTT. Therefore, most patients in this study were in their early stage of ACLF.

Our study had several limitations. First, although it included the largest cohort to date to undergo OGTTs to analyze the effects of the clinical characteristics of disturbances in glucose homeostasis and their associations with 90-day mortality in patients with HBV-related ACLF, it was a single-center study, and we could not perform OGTTs on some severe patients with ACLF. Thus, the number of patients included was relatively small, and the results should be verified in multicenter studies with more patients. Second, we evaluated only the association between baseline hyperglycemia and mortality, and whether the change in glucose metabolism disorders after admission and the different methods for managing hyperglycemia influence patient outcomes remains to be clarified.

In conclusion, this was the first study to evaluate the clinical characteristics of disturbances in glucose homeostasis and their associations with 90-day mortality in patients with HBV-related ACLF. The major finding in this study was that diabetes, but not hypoglycemia, is associated with high mortality in patients with ACLF.

Supplementary Information The online version contains supplementary material available at https://doi.org/10.1007/s12072-021-10243-1.

Author contributions Study concept and design: SL. Data collection: HH, XH, CT, YZ, QC, YL, FY. Statistical analysis: SL, HH, JL. Drafting the manuscript: $\mathrm{SL}, \mathrm{HH}, \mathrm{XH}$. Critical revision of the manuscript: JL, YL. All authors had access to the study data and reviewed and approved the final manuscript.

Funding This work was supported by the Chinese National Natural Science Foundation Project (81860114).

Data availability The data that support the findings of this study are available from the corresponding author upon reasonable request.

\section{Declarations}

Conflict of interest $\mathrm{Han} \mathrm{Hu}$, Xinxin Hu, Caiyun Tian, Yanping Zhu, Yujuan Liu, Qijiao Cheng, Fangwan Yang, Jun Liu, Ying Li and Shide Lin declare no competing interests.

Ethical approval The protocol of this study conformed to the tenets of the Declaration of Helsinki and was approved by the Human Ethical Committee of the Affiliated Hospital of Zunyi Medical University.

Informed consent Informed consent was obtained from all study participants.

Animal research This article does not contain any studies with animal subjects.
Consent to publish All authors approved the final version of the article including the authorship list and consented to publication in Hepatology International.

Plant reproducibility It is not applicable.

Clinical trials registration It is a retrospectively study and not registered.

\section{References}

1. Hamed AE, Elwan N, Naguib M, Elwakil R, Esmat G, El Kassas M, Abd-Elsalam S, et al. Diabetes association with liver diseases: an overview for clinicians. Endocr Metab Immune Disord Drug Targets. 2019;19:274-80.

2. Orsi E, Grancini V, Menini S, Aghemo A, Pugliese G. Hepatogenous diabetes: Is it time to separate it from type 2 diabetes? Liver Int. 2016;37:950-62.

3. Kushimoto S, Abe T, Ogura H, Shiraishi A, Saitoh D, Fujishima $\mathrm{S}$, Mayumi $\mathrm{T}$, et al. Impact of blood glucose abnormalities on outcomes and disease severity in patients with severe sepsis: an analysis from a multicenter, prospective survey of severe sepsis. PLoS ONE. 2020;15:e0229919.

4. Li H, Tian S, Chen T, Cui Z, Shi N, Zhong X, Qiu K, et al. Newly diagnosed diabetes is associated with a higher risk of mortality than known diabetes in hospitalized patients with COVID-19. Diabetes Obes Metab. 2020;22:1897-906.

5. Kim EJ, Jeong MH, Kim JH, Ahn TH, Seung KB, Oh DJ, Kim HS, et al. Clinical impact of admission hyperglycemia on in-hospital mortality in acute myocardial infarction patients. Int J Cardiol. 2017;236:9-15.

6. Dungan KM, Braithwaite SS, Preiser J-C. Stress hyperglycaemia. Lancet. 2009;373:1798-807.

7. Kumar R, Mehta G, Jalan R. Acute-on-chronic liver failure. Clin Med (Lond). 2020;20:501-4.

8. Ren Y, Liu L, Li Y, Yang F, He Y, Zhu Y, Hu X, et al. Development and validation of a scoring system to predict progression to acute-on-chronic liver failure in patients with acute exacerbation of chronic hepatitis B. Hepatol Res. 2018;48:692-700.

9. Imazeki F, Yokosuka O, Fukai K, Kanda T, Kojima H, Saisho $\mathrm{H}$. Prevalence of diabetes mellitus and insulin resistance in patients with chronic hepatitis $\mathrm{C}$ : comparison with hepatitis $\mathrm{B}$ virus-infected and hepatitis $C$ virus-cleared patients. Liver Int. 2008:28:355-62.

10. Bernal W, Wendon J. Acute liver failure. N Engl J Med. 2013;369:2525-34.

11. Vilstrup H, Iversen J, Tygstrup N. Glucoregulation in acute liver failure. Eur J Clin Invest. 1986;16:193-7.

12. Lee YS, Min KH, Lee SY, Shim JJ, Kang KH, Cho WH, Jeon D, et al. The value of glycated hemoglobin as predictor of organ dysfunction in patients with sepsis. PLoS ONE. 2019;14:e0216397.

13. Sigal SH, Stanca CM, Kontorinis N, Bodian C, Ryan E. Diabetes mellitus is associated with hepatic encephalopathy in patients with HCV cirrhosis. Am J Gastroenterol. 2006;101:1490-6.

14. Konishi I, Hiasa Y, Shigematsu S, Hirooka M, Furukawa S, Abe M, Matsuura B, et al. Diabetes pattern on the $75 \mathrm{~g}$ oral glucose tolerance test is a risk factor for hepatocellular carcinoma in patients with hepatitis C virus. Liver Int. 2009;29:1194-201.

15. Erice E, Llop E, Berzigotti A, Abraldes JG, Conget I, Seijo S, Reverter E, et al. Insulin resistance in patients with cirrhosis and portal hypertension. Am J Physiol Gastrointest Liver Physiol. 2012;302:G1458-1465 
16. Elkrief L, Chouinard P, Bendersky N, Hajage D, Larroque B, Babany G, Kutala B, et al. Diabetes mellitus is an independent prognostic factor for major liver-related outcomes in patients with cirrhosis and chronic hepatitis C. Hepatology. 2014;60:823-31.

17. Bianchi G, Marchesini G, Zoli M, Bugianesi E, Fabbri A, Pisi E. Prognostic significance of diabetes in patients with cirrhosis. Hepatology. 1994;20:119-25.

18. Hagel S, Bruns T, Herrmann A, Stallmach A, Schmidt C. Abnormal glucose tolerance: a predictor of 30-day mortality in patients with decompensated liver cirrhosis. Z Gastroenterol. 2011;49:331-4.

19. Sarin SK, Choudhury A, Sharma MK, Maiwall R, Al Mahtab M, Rahman S, Saigal S, et al. Acute-on-chronic liver failure: consensus recommendations of the Asian Pacific association for the study of the liver (APASL): an update. Hepatol Int. 2019;13:353-90.

20. Tsochatzis EA, Bosch J, Burroughs AK. Liver cirrhosis. Lancet. 2014;383:1749-61.

21. Bajaj JS, O'Leary JG, Reddy KR, Wong F, Olson JC, Subramanian RM, Brown G, et al. Second infections independently increase mortality in hospitalized patients with cirrhosis: the North American consortium for the study of end-stage liver disease (NACSELD) experience. Hepatology. 2012;56:2328-35.

22. Handelsman Y, Bloomgarden ZT, Grunberger G, Umpierrez G, Zimmerman RS, Bailey TS, Blonde L, et al. American association of clinical endocrinologists and American College of Endocrinology - clinical practice guidelines for developing a diabetes mellitus comprehensive care plan - 2015. Endocr Pract. 2015;21(Suppl 1):1-87.

23. Levy JC, Matthews DR, Hermans MP. Correct homeostasis model assessment (HOMA) evaluation uses the computer program. Diabetes Care. 1998;21:2191-2.

24. Grancini V, Trombetta M, Lunati ME, Zimbalatti D, Boselli ML, Gatti S, Donato MF, et al. Contribution of $\beta$-cell dysfunction and insulin resistance to cirrhosis-associated diabetes: role of severity of liver disease. J Hepatol. 2015;63:1484-90.

25. Mifsud S, Schembri EL, Gruppetta M. Stress-induced hyperglycaemia. Br J Hosp Med (Lond). 2018;79:634-9.

26. Ruan Y, Tan GD, Lumb A, Rea RD. Importance of inpatient hypoglycaemia: impact, prediction and prevention. Diabet Med. 2019;36:434-43.

27. Schattenberg JM, Schuchmann M. Diabetes and apoptosis: liver. Apoptosis. 2009;14:1459-71.

28. Nishida T, Tsuji S, Tsujii M, Arimitsu S, Haruna Y, Imano E, Suzuki M, et al. Oral glucose tolerance test predicts prognosis of patients with liver cirrhosis. Am J Gastroenterol. 2006;101:70-5.

29. Lai RM, Chen TB, Hu YH, Wu G, Zheng Q. Effect of type 2 diabetic mellitus in the prognosis of acute-on-chronic liver failure patients in China. World J Gastroenterol. 2021;27:3372-85.

30. Duseja A, De A, Taneja S, Choudhury AK, Devarbhavi H, Hu J, Hamid SS, et al. Impact of metabolic risk factors on the severity and outcome of patients with alcohol-associated acute-on-chronic liver failure. Liver Int. 2021;41:150-7.

31. Kappus MR. Acute hepatic failure and nutrition. Nutr Clin Pract. 2020;35:30-5.

32. Pfortmueller CA, Wiemann C, Funk GC, Leichtle AB, Fiedler GM, Exadaktylos AK, Lindner G. Hypoglycemia is associated with increased mortality in patients with acute decompensated liver cirrhosis. J Crit Care. 2014;29(316):e317-e312.

Publisher's Note Springer Nature remains neutral with regard to jurisdictional claims in published maps and institutional affiliations. 